\title{
Soluble Thrombomodulin and Major Orthopedic Surgery
}

\author{
Irina P. Antropova, $\mathrm{PhD}, \mathrm{ScD}^{1,2 *}$; Boris G. Yushkov, $\mathrm{PhD}, \mathrm{ScD}^{3,4}$; \\ Evgeny Kobrinsky, $\mathrm{PhD}^{5}$; Anatoliy N. Varaksin, $\mathrm{PhD}, \mathrm{ScD}^{6}$ \\ ${ }^{1}$ Ural State Medical University, Ekaterinburg, Russia \\ ${ }^{2}$ Ural Institute of Traumatology and Orthopedics named after V.D. Chaklin, Ekaterinburg, Russia \\ ${ }^{3}$ Institute of Immunology and Physiology, the Urals Branch of the RAS, Ekaterinburg, Russia \\ ${ }^{4}$ Ural Federal University named after the first President of Russia B.N.Yeltsin, Ekaterinburg, Russia \\ ${ }^{5}$ National Institute on Aging, NIH, Baltimore, MD, USA \\ ${ }^{6}$ Institute of Industrial Ecology, the Urals Branch of the RAS, Ekaterinburg, Russia
}

\begin{abstract}
Background: A high level of soluble thrombomodulin (sTM) is associated with a lower risk of thrombosis but can cause severe bleeding after operations. Deep vein thrombosis (DVT) and blood loss are serious threats after orthopedic surgery. The aim of our pilot study was to evaluate the effect of the preoperative level of sTM on coagulation and inflammation as well as the blood loss and the development of symptomatic DVT after total large joint replacement.

Methods and Results: In all patients $(\mathrm{n}=50)$ who underwent total hip or knee replacement, sTM, PrC, D-dimer, vWF, CRP, and platelets were determined before and after the operation. According to the preoperative sTM level, patients were divided into 2 groups: the thrombomodulin low (TML) group $(n=25)$ and thrombomodulin high $(\mathrm{TMH})$ group $(\mathrm{n}=25)$. The concentration of sTM was $4.4[3.4,4.7] \mathrm{ng} / \mathrm{ml}$ in the TML-group and $8.7[7.3,10.6] \mathrm{ng} / \mathrm{ml}$ in the TMH-group. After surgery, D-dimer, vWF, platelet count and CRP were higher and total blood loss was lower in the TML group. In the TML-group, a symptomatic DVT was detected in $3(12 \%)$ patients; in the TMH-group, a symptomatic DVT was identified only in 1(4\%) case.

Conclusion: These findings support the important role of sTM in coagulation, inflammation, bleeding, and presumably in venous thrombosis after major orthopedic surgery. (Int J Biomed. 2016;6(3):213-217.).
\end{abstract}

Key Words: thrombomodulin • coagulation • bleeding • total hip and knee replacement.

\section{Abbreviations}

CRP, C-reactive protein; DVT, deep vein thrombosis; IQR, interquartile range; PrC, protein C; TM, thrombomodulin; sTM, soluble TM; vWF, von Willebrand factor.

\section{Introduction}

Human TM is a single-chain, type 1 transmembrane glycoprotein mainly expressed on the luminal surface of the vascular endothelium. ${ }^{[1]} \mathrm{TM}$ binds thrombin and modifies its

*Corresponding author: Irina Antropova, PhD, ScD. Ural State Medical University, Ural Institute of Traumatology and Orthopedics named after V.D. Chaklin; Ekaterinburg, Russia.

E-mail: aip.hemolab@mail.ru conformation, which allows activation of $\operatorname{PrC}{ }^{\left[{ }^{[2]}\right.}$ In addition, TM plays an important role in mediating anti-inflammatory activity. ${ }^{[3]}$ Plasma sTM is formed by proteolysis of membranebound $\mathrm{TM}^{[4]}$ with anticoagulant and anti-inflammatory properties. ${ }^{[5]}$ Experimental and clinical studies have shown that a low level of sTM is associated with a prethrombotic state and venous thrombosis. ${ }^{[6-9]}$ Application of recombinant human sTM is effective in prevention of the venous thrombosis in a rat model and after total hip replacement ${ }^{[10,11]}$ for the treatment of disseminated intravenous coagulation. ${ }^{[12]}$ On the other 
hand, the increased level of sTM causes severe bleeding after injuries and operations. ${ }^{[13]}$

Thromboembolic complications and increased blood loss are serious threats after major orthopedic surgery. ${ }^{[14]}$ The incidence of venography-detected DVT ranges from $42 \%$ to $57 \%$ in total hip replacement and $41 \%$ to $85 \%$ in total knee replacement, in the absence of prophylaxis. ${ }^{[15]}$

The aim of our pilot study was to evaluate the effect of the preoperative level of sTM on coagulation and inflammation as well as the blood loss and the development of symptomatic DVT after total large joint replacement.

\section{Materials and Methods}

The study included 50 patients $(22$ men, aged from 30 to 74 years) admitted for total hip or knee replacement. Exclusion criteria were coagulopathy and anticoagulant or antiplatelet therapy before surgery. The study was conducted in accordance with ethical principles of the Declaration of Helsinki and approved by the local Ethics Committees. Written informed consent was obtained from each patient.

All patients received antithrombotic prophylaxis with enoxaparin (40 mg daily), first administered in the evening before the operation, and then administered daily for two weeks. In order to limit blood loss, all patients included in the study received tranexamic acid $(15-20 \mathrm{mg} / \mathrm{kg}$ intravenously). The first dose was administered 20-30 minutes before the surgical incision and the second dose was administered 6 hours after the first infusion. Intraoperative and postoperative bleeding was monitored. Clinical parameters indicative of thrombosis were recorded, and patients with suspected DVT were admitted for Doppler ultrasound verification.

All patients were divided into 2 groups according to the preoperative sTM level (Fig.1). The median value for sTM was $5.6 \mathrm{ng} / \mathrm{mL}$. The data obtained from patients with sTM level below the median was combined into a thrombomodulin low (TML) group $(n=25)$; the data obtained from patients with sTM levels equal to or above the median made up the thrombomodulin high (TMH) group $(\mathrm{n}=25)$. The concentration of sTM was $4.4[3.4,4.7] \mathrm{ng} / \mathrm{ml}$ in the TML-group and $8.7[7.3$, $10.6] \mathrm{ng} / \mathrm{ml}$ in the TMH-group. The normal reference range was $5.1[2.9,7.6] \mathrm{ng} / \mathrm{ml}$, according to the manufacturer of the reagents. Characteristics of patients and surgical procedures in selected groups are presented in Table 1. The age of patients in the TMH-group was higher than in the TML-group, and this fact was taken into account when carrying out a statistical analysis of research results.

Blood sampling was performed at set time $(\mathrm{T})$ points during the perioperative period: preoperatively 1 to 2 days (T1), 30 minutes after completing the replacement (T2), postoperative Day 1 (T3), Day 3 (T4), Day 7 (T5), and Day 14 (T6). Venous blood samples were collected in the morning into a test tube containing a $3.2 \%$ sodium citrate solution in a ratio of 1:9 sodium citrate/blood. Platelet count was determined in EDTA-stabilized blood. Blood serum was used for quantitative determination of C-reactive protein.

The following analyses were carried out for all patients: TM («Human sCD141 ELISA Kit», Gen Probe Diaclone,
France); PrC («Technozym Protein C ELISA», Technoclone, Austria); D-dimer («Technozym D-dimer ELISA», Technoclone, Austria); von Willebrand factor («Technozym vWF: Ag ELISA», Technoclone, Austria), platelet count. C-reactive protein ( $«$ High Sensitivity C-Reactive Protein Enzyme Immunoassay Kit», Biomerica, Germany) was determined for 38 patients. All analyses were performed in accordance with the manufacturerss instructions.
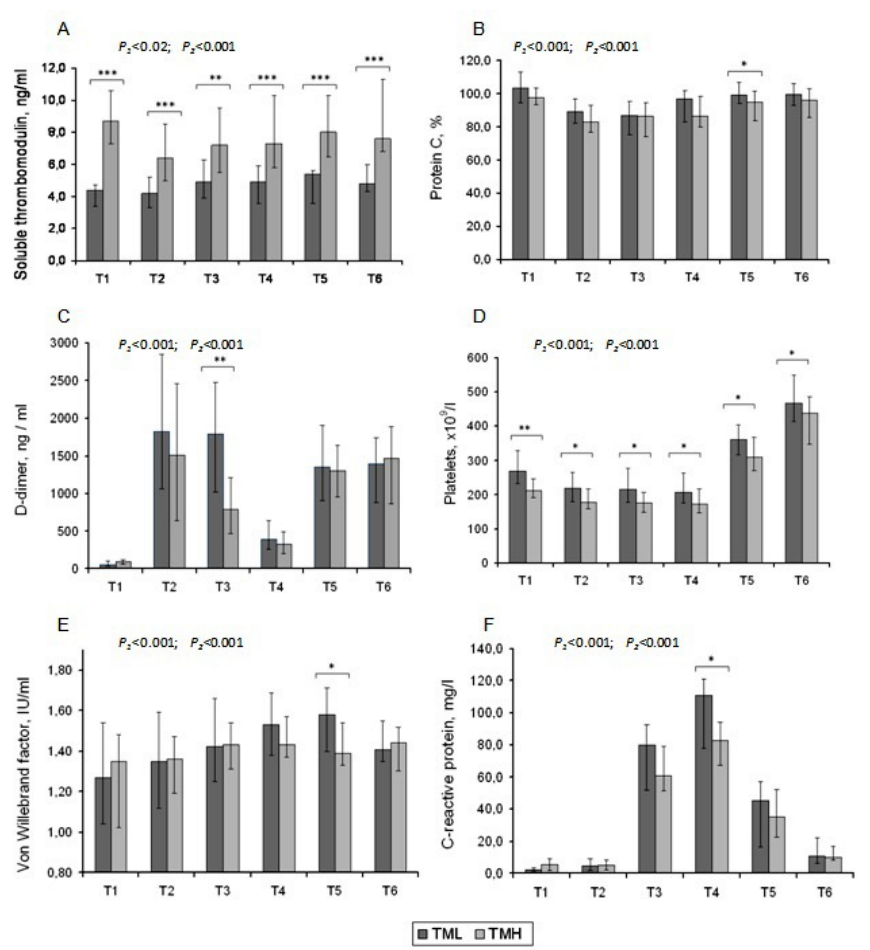

Fig 1. Coagulation and inflammation parameters before and after total large joints replacement.

Differences in concentrations and activity of STM (A), PrC (B), D dimer $(C)$, platelet count $(D), v W F(E), C R P(F)$ for $T M L$ and TMH groups are shown in histogram $\left(*_{-} P<0.05\right.$; $\left.*_{*}-P<0.01 ; * * *_{-} P<0.001\right)$. P1 - statistical significance of postoperative changes for the TML-group; P2 - statistical significance of postoperative changes for the TMH-group.

Table 1.

Baseline characteristics of patients and surgical aspects

\begin{tabular}{|c|c|c|c|}
\hline Variable & TML group & TMH group & $P$ \\
\hline Gender (male/female), No (\%) & $10 / 15(40 / 60)$ & $12 / 13(48 / 52)$ & 0.573 \\
\hline Age, years, median [IQR] & $51[47,63]$ & $62[57,65]$ & 0.032 \\
\hline $\begin{array}{l}\text { Type of surgery } \\
\text { (THR/TKR), No. (\%) }\end{array}$ & $21 / 4(84 / 16)$ & $20 / 5(80 / 20)$ & 0.818 \\
\hline $\begin{array}{l}\text { Prostheses } \\
\text { (cementless/cemented), No.(\%) }\end{array}$ & $19 / 6(76 / 24)$ & $15 / 10(60 / 40)$ & 0.335 \\
\hline $\begin{array}{l}\text { Duration of surgery, } \\
\text { min, median [IQR] }\end{array}$ & $100[85,110]$ & $100[90,120]$ & 0.397 \\
\hline $\begin{array}{l}\text { Type of anesthesia } \\
\text { (total/regional ), No.(\%) }\end{array}$ & $10 / 15(40 / 60)$ & $6 / 19(24 / 76)$ & 0.335 \\
\hline
\end{tabular}

THR - total hip replacement, TKR - total knee replacement. The results are expressed as a Number (percentage) and Median [IQR]. 
Statistical differences between two groups were analyzed by nonparametric Mann Whitney $U$ test; the differences between time points T1 - T6 - by Friedman's ANOVA by ranks. A probability value of $P<0.05$ was considered statistically significant. The effect of age was analyzed by regression analysis and analysis of covariance. The results are expressed as Median [interquartile range (IQR)].

\section{Results and Discussion}

We observed that the patients with higher levels of sTM were older. A similar association between sTM and age was found earlier. ${ }^{[16]}$ Blood sTM level is determined by hereditary factors ${ }^{[9,13]}$ and by pathological changes that lead to increased proteolytic cleavage of endothelial TM. ${ }^{[17]} \mathrm{TM}$ expression is depressed in the aged. ${ }^{[18]}$ On the other hand, aging is often accompanied by increasing endothelial dysfunction; ${ }^{[19]}$ it can enhance the shedding of the soluble fragments of TM into circulating blood. Regression analysis showed that in the TMH-group, the patients' age affects only the preoperative level of PrC and CRP, as well as the platelet count at day 7 after the operation. After analysis of covariance, results for the dependent parameters were adjusted.

sTM level in the TML-group increased by Day 1 after surgery and stayed above the initial level until Day 14. On the contrary, in the TMH-group, TM level decreased after the operation and then was gradually restored $(P<0.02$ and $P<0.001$ respectively in Friedman's ANOVA, Fig. 1A). Despite the decline in the level of sTM in the TMH-group, significant differences in sTM levels between the groups were observed throughout the study period, and we can conclude that the postoperative level of sTM depends on its preoperative level.

PrC levels in both groups significantly decreased after the operation, and then gradually recovered $(P<0.001$ in Friedman's ANOVA for both; Fig. 1B). We observed a tendency to a lower level of $\operatorname{PrC}$ in the TMH-group compared with the TML-group throughout the study period. The observed differences were statistically significant by Day $7(P=0.034)$. We cannot exclude that when there is a decrease in the liver production of $\mathrm{PrC}$, an increase of the TM cleavage may have a compensatory effect for the anticoagulant pathways.

The concentration of D-dimer in TML-group was greatly increased immediately after surgery and Day 1 after the joint replacement. We observed a significant shift towards normalization in the concentration of D-dimer by postoperative Day 3, which was followed by another increase in the D-dimer level $(P<0.001$ in Friedman's ANOVA for both; Fig. 1C). The TMH-group had a more rapid decrease in the activity of coagulation after the operation. The concentration of D-dimer was substantially lower in this group than in the TML-group by Day 1 after surgery $(P=0.002)$. Soluble TM fragments have full cofactor activity and the ability to bind thrombin and activate $\operatorname{PrC} .{ }^{[1]} \mathrm{A}$ more rapid decrease of the $\mathrm{D}$-dimer level is apparently due to more effective inactivation of the thrombin in patients with high levels of TM. We can assume that higher levels of sTM provide a more effective inhibition of coagulation induced by total large joint replacement.
The platelet count performed before surgery in the TMHgroup was significantly lower than in the TML-group. After a total large joint replacement in both groups, the platelet count decreased for 3 days followed by a gradual increase exceeding the preoperative level $(P<0.001$ in Friedman's ANOVA for both; Fig. 1D). Significant differences between the groups remained in the postoperative period. A negative relationship between platelet count and sTM level was found in both healthy persons and those with pathological conditions. ${ }^{[16,20]}$ We observed a similar relationship in our study before and after major orthopedic surgery.

vWF is considered to be one of the known endothelial markers, and changes in its concentration reflect the degree of the inflammatory response after large joint replacement. ${ }^{[2]} \mathrm{We}$ observed a gradual increase in vWF over time in both groups $(P<0.001$ in Friedman's ANOVA for both; Fig. 1E). However, the increase in vWF was less prominent in the TMH-group than in the TML-group, and these differences reached statistical significance by Day $7(P=0.032)$.

The serum level of CRP is an indicator of the acute phase inflammatory response and can be used as a monitoring tool. ${ }^{[22]}$ The concentration of CRP was significantly increased after surgery, reaching maximum values by Day 3 . After that, the CRP level had a tendency to normalize but was never able to reach the baseline during the study period $(P<0.001$ in Friedman's ANOVA for both; Fig. 1F). CRP dynamics showed a similar character in both groups; however, the maximum CRP level was significantly lower in the TMH-group than in the TML-group $(P=0.019)$. TM has been shown to mediate antiinflammatory activities using activated protein $\mathrm{C}$-dependent and activated protein $\mathrm{C}$-independent mechanisms. ${ }^{[3]}$ In our study, a higher level of sTM was associated with less increase of CRP and vWF after surgery. We concluded that sTM reduces the intensity of the inflammatory response after a total large joint replacement.

Intraoperative and postoperative bleeding monitoring has shown that the total blood loss (Intraoperative and postoperative within 24 hours) was significantly higher in the TMH-group than in the TML-group (Fig.2).

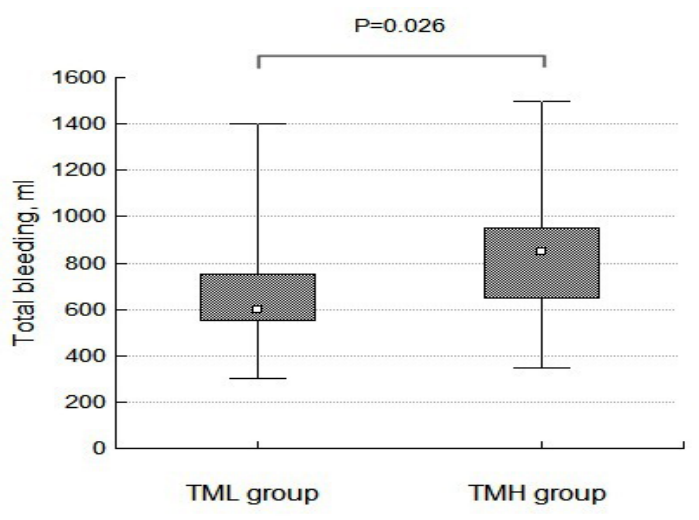

Fig. 2. Total bleeding ( $\mathrm{ml}$ ) after a total large joint replacement.

The box represents the median and IQR, the whiskers represents the minimum and maximum, $P=0.026$. 
There were no significant differences in the localization of the operated segment, the duration of surgery, and the type of anesthesia between groups with high and low levels of sTM (Table 1). However, the blood loss was significantly greater in patients with higher sTM levels. Apparently, a high sTM level promotes rapid activation of $\operatorname{PrC}$ and early interruption of thrombin generation. This worsens the propagation phase of coagulation and increases blood loss. Our results are in line with the observation of Y. Dargaud et al. (2015); they have shown that increased levels of sTM cause severe bleeding after injuries and operations. ${ }^{[13]}$

Platelets play a critical role in hemostasis during vessel damage. In our study, the high sTM level was associated with a relatively low platelet count in the perioperative period, which suggests that a synergy of these factors contributes to the blood loss.

Previous experimental studies suggest that TM deficiency is associated with the prethrombotic state. ${ }^{[6,7]}$ Significantly lower levels of plasma sTM were detected in patients with thrombosis in comparison with healthy individuals. ${ }^{[8]} \mathrm{We}$ have detected less venous thrombosis after total large joint replacement in patients who have a higher sTM level before and after surgery. In the TML-group, a symptomatic DVT was detected in 3(12\%) patients; in the TMH-group, a symptomatic DVT was identified only in $1(4 \%)$ case. Although the differences did not reach statistical significance, our results do not contradict previously published data.

Our study has some limitations: a relatively small number of patients; participants were divided into groups retrospectively according to the median of the preoperative sTM level; connection of sTM with age; Doppler ultrasound was used only for confirming a symptomatic DVT, so we could not detect a "silent" thrombosis. Despite these limitations, we assume the obtained results support a link between the variation in preoperative STM level and the activity of coagulation and inflammation after total large joint replacement. Further research may help in preoperative preparation and postoperative treatment of patients who require major orthopedic surgery.

\section{Acknowledgements}

We thank all physicians of the Ural Institute of Traumatology and Orthopedics named after V.D. Chaklin for their participation in this study.

\section{Sources of Funding}

This study was supported by the research program of the Russian Federation № 215063040014 and in part by the Intramural research Program of the NIH, National Institute on Aging (USA).

\section{Competing interests}

The authors declare that they have no competing interests.

\section{References}

1. Tsiang M, Lentz SR, Sadler JE. Functional domains of membrane-bound human thrombomodulin. EGF-like domains four to six and the serine/threonine-rich domain are required for cofactor activity. J Biol Chem. 1992; 267(9):6164-70.

2. Weiler H, Isermann BH. Thrombomodulin. J Thromb Haemost. 2003;1(7):1515-24.

3. Conway EM. Thrombomodulin and its role in inflammation. Semin Immunopathol. 2012; 34(1):107-25.

4. Menschikowski M, Hagelgans A, Eisenhofer G, Tiebel O, Siegert G. Reducing agents induce thrombomodulin shedding in human endothelial cells. Thromb Res. 2010;126(2):e88-93. 5. Ohlin AK, Larsson K, Hansson M. Soluble thrombomodulin activity and soluble thrombomodulin antigen in plasma. J Thromb Haemost. 2005;3(5):976-82.

6. Weiler-Guettler H, Christie PD, Beeler DL, Healy AM, Hancock WW, Rayburn H, et al. A targeted point mutation in thrombomodulin generates viable mice with a prethrombotic state. J Clin Invest. 1998;101(9):1983-91.

7. Raife TJ, Dwyre DM, Stevens JW, Erger RA, Leo L, Wilson KM, et al. Human thrombomodulin knock-in mice reveal differential effects of human thrombomodulin on thrombosis and atherosclerosis. Arterioscler Thromb Vasc Biol. 2011;31(11):2509-17.

8. Javanmard SH, Shahsavarzadeh T, Saadatnia M. Soluble thrombomodulin and endothelial cell protein $\mathrm{C}$ receptor levels in patients with cerebral venous and sinus thrombosis. Eur Neurol. 2013;70(3-4):156-8.

9. Navarro S, Medina P, Bonet E, Corral J, Martínez-Sales $\mathrm{V}$, Martos L, et al. Association of the thrombomodulin gene c. $1418 \mathrm{C}>\mathrm{T}$ polymorphism with thrombomodulin levels and with venous thrombosis risk. Arterioscler Thromb Vasc Biol. 2013;33(6):1435-40.

10. Solis MM, Cook C, Cook J, Glaser C, Light D, Morser J, et al. Intravenous recombinant soluble human thrombomodulin prevents venous thrombosis in a rat model. J Vasc Surg. 1991;14(5):599-604.

11. Kearon C, Comp P, Douketis J, Royds R, Yamada K, Gent M. Dose-response study of recombinant human soluble thrombomodulin (ART-123) in the prevention of venous thromboembolism after total hip replacement. J Thromb Haemost. 2005;3(5):962-8.

12. Aota T, Wada H, Yamashita Y, Matsumoto T, Ohishi $\mathrm{K}$, Suzuki K, et al. The efficacy of the administration of recombinant human soluble thrombomodulin in patients with DIC. Int J Hematol. 2016;103(2):173-9.

13. Dargaud Y, Scoazec JY, Wielders SJ, Trzeciak C, Hackeng TM, Négrier C, et al. Characterization of an autosomal dominant bleeding disorder caused by a thrombomodulin mutation. Blood. 2015;125(9):1497-501.

14. Falck-Ytter Y, Francis CW, Johanson NA, Curley C, Dahl OE, Schulman S, et al. Prevention of VTE in orthopedic surgery patients: Antithrombotic Therapy and Prevention of Thrombosis, 9th ed: American College of Chest Physicians Evidence-Based Clinical Practice Guidelines. Chest. 2012; 141(2 Suppl): e278S-325S.

15. Lowe GD. Prediction of postoperative deep-vein thrombosis. Thromb Haemost 1997;78(1):47-52.

16. Randi ML, Meneghin C, Zerbinati P, Sbarai A, Rampin E, Pasini R, et al. Soluble plasma thrombomodulin levels in patients with chronic myeloproliferative disorder. Clin Appl Thromb Hemost. 1999;5(1):43-7. 
17. Takano S, Kimura S, Ohdama S, Aoki N. Plasma thrombomodulin in health and diseases. Blood. 1990; 76(10):2024-9.

18. Starr ME, Ueda J, Takahashi H, Weiler H, Esmon CT, Evers BM, Saito H. Age-dependent vulnerability to endotoxemia is associated with reduction of anticoagulant factors activated protein C and thrombomodulin. Blood. 2010;115(23):4886-93. 19. Abbate R, Prisco D, Rostagno C, Boddi M, Gensini GF. Age-related changes in the hemostatic system. Int J Clin Lab Res. 1993;23(1):1-3.

20. Osada M, Kaneko M, Sakamoto M, Endoh M, Takigawa
$\mathrm{K}$, Suzuki-Inoue $\mathrm{K}$, et al. Causes of thrombocytopenia in chronic hepatitis $\mathrm{C}$ viral infection. Clin Appl Thromb Hemost. 2012;18(3):272-80.

21. Hughes SF, Hendricks BD, Edwards DR, Maclean KM, Bastawrous SS, Middleton JF. Total hip and knee replacement surgery results in changes in leukocyte and endothelial markers. J Inflamm (Lond). 2010;7:2.

22. Roberts W.L, Sedrick R, Moulton L, Spencer A, Rifai N. Evaluation of four automated high-sensitivity C-reactive protein methods: implications for clinical and epidemiological applications. Clin.Chem 2000;46(4):461-8. 